\title{
HARDY SPACE EXPECTATION OPERATORS AND REDUCING SUBSPACES
}

\author{
JOSEPH A. BALL
}

\begin{abstract}
In this paper we study the range of the isometry on $H^{p}$ arising from an inner function which is zero at zero by composition. The range of such an isometry is characterized as a closed subspace of $H^{p}$ (weak-* closed for $p=\infty$ ) satisfying the following: (i) the constant function 1 is in $\pi$; (ii) if $f \in \mathbb{M}$ and $g \in H^{\infty} \cap \pi$, then $f g \in \mathbb{\pi}$; (iii) if $f \in \pi$ has inner-outer factorization $f=x \cdot F$, then $x$ is in $\pi$; (iv) if $\left\{B_{a}: a \in \mathbb{Q}\right\}$ is a collection of inner functions in $\pi$, then the greatest common

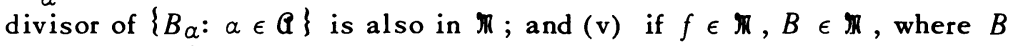
is inner and $\bar{B} \cdot f \in H^{p}$, then $\bar{B} \cdot f \in M$. The proof makes use of the fact that there exists a projection onto such a subspace satisfying the axioms of an expectation operator, which for $p=2$, is simply the orthogonal projection. This characterization is applied to give an equivalent formulation of a conjecture of Nordgren concerning reducing subspaces of analytic Toeplitz operators.
\end{abstract}

1. Introduction. Let $L^{p}$ be the space of Lebesgue measurable functions on the circle whose pth power is integrable, and let $H^{p}$ be those elements of $L^{p}$ whose negative Fourier coefficients vanish. For $\phi$ an inner function with $\phi(0)=0$, define the operator $C_{\phi}$ on $L^{p}$ by

$$
C_{\phi}: f\left(e^{i \theta}\right) \rightarrow f\left(\phi\left(e^{i \theta}\right)\right) \text {. }
$$

This operator, studied by Nordgren [7] and Ryff [ 10] among others, has $H^{p}$ as an invariant subspace, and under our assumptions on $\phi$, is an isometry. Our main result is a characterization of the range of $C_{\phi}$ considered as an operator on $H^{p}, 1 \leq p \leq \infty$. See Hoffman [ 5] or Sz.-Nagy-Foiaş [12] for relevant definitions. The notation g.c.d. means greatest common divisor.

Theorem 1. A closed $\left(p=\infty\right.$, weak- ${ }^{*}$ closed) subspace $\mathbb{M}$ of $H^{p}$ is the range of an operator $C_{\phi}$ for some inner $\phi$ with $\phi(0)=0$ if and only if.

(i) $1 \in \mathbb{M}, \mathbb{M}$ contains a nonconstant function;

(ii) if $f \in \mathbb{M}$ and $g \in H^{\infty} \cap \mathbb{M}$, then $f g \in \mathbb{M}$;

Received by the editors November 26, 1973.

AMS (MOS) subject classifications (1970). Primary 47B35, 46J15. 
(iii) if $f \in \mathbb{M}$ has inner-outer factorization $f=\chi \cdot F$, then $\chi \in M$ and $F \in \mathbb{M}$;

(iv) if $\left\{B_{\alpha} \mid \alpha \in \mathbb{Q}\right\}$ is a collection of inner functions in $\mathbb{M}$, then $B=$ g.c.d. $\left\{B_{a}\right\}$ is in $\mathbb{M}$;

(v) if $f \in \mathbb{M}, B \in \mathbb{M}$, where $B$ is inner and $\bar{B} f \in H^{p}$, then $\bar{B} \cdot f \in \mathbb{M}$.

2. Expectation operators associated with $\phi$. Let $\mathscr{B}$ be the smallest $\sigma$-algebra of Lebesgue measurable sets with respect to which $\phi$ is measurable. Given any $f$ in $L^{p}$, by the Radon-Nikodym theorem there exists a unique $B$-measurable function $\left(P_{\phi} f\right)$ such that $\int_{B} f d m=\int_{B}\left(P_{\phi} f\right) d m$ for all $B \in \Re$. The function $\left(P_{\phi} f\right)$ is the Radon-Nikodym derivative of $f$ with re-

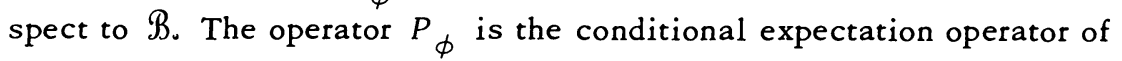
probability theory and has the following properties [6]:

(2) $P_{\phi}(1)=1$;

(3) $P_{\phi}\left(f \cdot P_{\phi}(g)\right)=\left(P_{\phi} f\right)\left(P_{\phi} g\right)$;

(4) $\left\|P_{\phi} f\right\|_{p} \leq\|f\|_{p}$, that is, $\mathrm{P}_{\phi}$ is a contraction operator on $L^{p}$;

(5) $P_{\phi}$ is a projection, $P_{\phi}^{2}=P_{\phi}$;

(6) $\int\left(P_{\phi} f\right) \cdot g d m=\int f\left(P_{\phi} g\right) d m$ for $f \in L^{p}, g \in L^{q}, 1 / p+1 / q=1$;

(7) the range of $P_{\phi}$ is $L^{p}(\Re)$, the set of functions in $L^{p}$ measurable with respect to $\Re$, where $P_{\phi}$ is considered as an operator on $L^{p}$.

We state the following Lemma adapted from Rota [9] without proof.

Lemma 1. Let $\mathbb{A}$ be the collection of all functions of the form $p(\phi, \bar{\phi})$, $p$ a complex polynomial in two variables. Then, for $1 \leq p<\infty, L^{p}(\Re)$ is the closure in $L^{p}$ of $\mathbb{A}$, and $L^{\infty}(\mathfrak{B})$ is the weak. ${ }^{*}$ closure in $L^{\infty}$ of $\mathbb{A}$.

We now use the special assumptions on $\phi$. Since $\phi$ is inner, $\bar{\phi}^{j} \phi^{k}=$ $\phi^{k-j}$, and a polynomial $p(\phi, \bar{\phi})$ in $\phi$ and $\bar{\phi}$ has the form $\Sigma_{j=-n}^{n} c_{j} \phi^{j}$. Using this we can obtain

Lemma 2. $P_{\phi}$ leaves $H^{p}$ invariant, $1 \leq p \leq \infty$.

Proof. Consider first $p=2$. Since $\phi$ is inner with $\phi(0)=0,\left\{\phi^{j}: j\right.$ an integer $\}$ is an orthonormal set in $L^{2}$. By Lemma 1 , this orthonormal set spans $L^{2}(\mathcal{B}) \equiv \operatorname{ran} P_{\phi}$. By (5) and (6), $P_{\phi}$ is the orthogonal projection onto

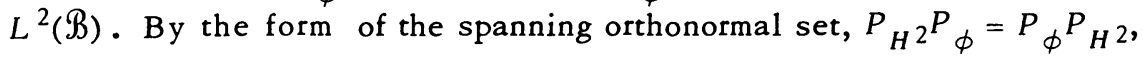
and the assertion follows for $p=2$.

Suppose $f \in H^{p}, 1 \leq p \leq \infty$. Then by (6), for all $g \in H_{0}^{\infty}, \int\left(P_{\phi} f\right) g d m=$ $\int f\left(P_{\phi} g\right) d m$. Since $g \in H_{0}^{\infty} \subset H_{0}^{2},\left(P_{\phi} g\right) \in H_{0}^{2} \cap L^{\infty}=H_{0}^{\infty}$, where the last integral vanishes since $f \in H^{p}$. Hence $P_{\phi} f \in H^{p}$. 
Lemma 3. $P_{\phi}$ is a projection of $L^{p}$ onto $C_{\phi}\left(L^{p}\right)$, and of $H^{p}$ onto $C_{\phi}\left(H^{p}\right)$.

Proof. For $1 \leq p<\infty$, use Lemma 1 , the fact that $\bar{\phi}^{j} \phi^{k}=\phi^{k-j}$, and that $C_{\phi}$ is an isometry. For $p=\infty$, also use the characterization of sequential weak- ${ }^{*}$ convergence as bounded point-wise convergence.

Notation. For convenience of notation, let us set

$$
L^{p}(\phi)=P_{\phi}\left(L^{p}\right)\left(=C_{\phi}\left(L^{p}\right)\right) \quad \text { and } \quad H^{p}(\phi)=P_{\phi}\left(H^{p}\right)\left(=C_{\phi}\left(H^{p}\right)\right) .
$$

The shift operator $S: H^{p} \rightarrow H^{p}$ defined by $S: f\left(e^{i \theta}\right) \rightarrow e^{i \theta} f\left(e^{i \theta}\right)$ is an isometry on $H^{p}$; the closed (for $p=\infty$, weak ${ }^{*}$ closed) invariant subspaces of $S$ have been characterized (for $p=2$, Beurling [1], for arbitrary $p$, Srinivasan and Wang [11]) as of the form $B \cdot H^{p}$, where $B$ is inner (i.e. , unimodular almost everywhere). If $p=2$ and $\pi$ is such an invariant subspace with the property that not all $f \in \Re$ have $f(0)=0$, a nonzero constant multiple of the associated inner function $B$ is obtained as the orthogonal projection of the constant function 1 onto $\pi$ (Hoffman $[5, \mathrm{p} .100])$. We use this fact in the proof of the next lemma.

Lemma 4. Let $K$ be a collection of functions in $H^{p}(\phi)$, and let the smallest closed (for $p=\infty$, weak ${ }^{*}$ closed) invariant subspace of $S$ containing $K$ be equal to $B \cdot H^{p}$, where $B$ is the associated inner function. Then $B \in H^{p}(\phi)$.

Proof. First consider the case $p=2$. Without loss of generality, we can assume that not all elements of $K$ vanish at the origin. Otherwise, let $j$ be the largest integer such that $K \subset \phi^{j} \cdot H^{2}(\phi)$. Then $K^{\prime}=\bar{\phi}^{j} K \subset$ $H^{2}(\phi)$ and not all elements of $K^{\prime}$ vanish at the origin. If the invariant subspace of $S$ generated by $K^{\prime}$ is $B^{\prime} \cdot H^{2}$ where $B^{\prime}$ is inner and in $H^{2}(\phi)$, then the invariant subspace generated by $K$ is simply $B \cdot H^{2}$, where $B=$ $\phi^{j} B^{\prime}$ is in $H^{2}(\phi)$.

Hence, assume not all elements of $K$ vanish at the origin, and let $\pi$ be the invariant subspace of $S$ generated by $K$. Note that finite sums of elements of the form $f \cdot k$, where $f \in H^{\infty}$ and $k \in K$, form a dense subset of $\Re$. Under our assumption, $c \cdot B, c$ some complex number, $0<|c| \leq 1$, is the orthogonal projection of the constant function 1 onto $\pi$. Hence, by elementary Hilbert space results,

$$
\|1-c \cdot B\|_{2}^{2}=\inf \left\{\|1-g\|_{2}^{2}: g=\sum_{i=1}^{N} f_{i} k_{i}, f_{i} \in H^{\infty}, k_{i} \in K\right\},
$$


and if $\left\{g_{n}\right\}$ is any minimizing sequence, $g_{n}$ converges to $c \cdot B$ in $H^{2}$. Note that, for $f_{i} \in H^{\infty}, k_{i} \in K$, using properties (2)-(4) of $P_{\phi}$.

$$
\begin{aligned}
\left\|1-\sum_{i=1}^{N} P_{\phi}\left(f_{i}\right) \cdot k_{i}\right\|_{2} & =\left\|1-P_{\phi}\left(\sum_{i=1}^{N} f_{i} \cdot k_{i}\right)\right\|_{2} \\
& =\left\|P_{\phi}\left(1-\sum_{i=1}^{N} f_{i} \cdot k_{i}\right)\right\|_{2} \leq\left\|1-\sum_{i=1}^{N} f_{i} \cdot k_{i}\right\|_{2} .
\end{aligned}
$$

Hence, if $\left\{\Sigma_{i=1}^{N_{n}} f_{i_{n}} \cdot k_{i_{n}}\right\}$ is a minimizing sequence, $\left\{\Sigma_{i=1}^{N} P_{\phi}\left(f_{i_{n}}\right) \cdot k_{i_{n}}\right\}$ $\subset \dot{H}^{2}(\phi)$ is also minimizing, $B=\lim _{n \rightarrow \infty} \sum_{i=1}^{N_{n}} P_{\phi}\left(f_{i_{n}}\right) \cdot k_{i_{n}}$ is in $H^{2}(\phi)$.

For $p \neq 2, p<\infty, f$ in $H^{p}$ having inner-outer factorization $f=\chi \cdot F$, note that $f \rightarrow f^{\prime}=\chi \cdot F^{p / 2}$ maps $H^{p}$ onto $H^{2}$ with $\|f\|_{p}^{p}=\left\|f^{\prime}\right\|_{2}^{2}$, and an invariant subspace closed in $H^{p}$ norm is mapped onto an invariant subspace closed in $H^{2}$ norm. For $p=\infty$, simply use that the closure of $B \cdot H^{\infty}$ in $H^{2}$-norm is $B \cdot H^{2}$. In this way the general situation is reduced to the case $p=2$.

3. Proof of Theorem 1. We first show necessity in Theorem 1, that is the subspace $H^{p}(\phi)$ for $\phi$ an inner function with $\phi(0)=0$, satisfies conditions (i) $-(v)$ in the statement of Theorem 1. (i) and (ii) are clear. For $(v)$, note that in $f \in H^{p}(\phi), B$ is inner and in $H^{p}(\phi)$ such that $\bar{B} f \epsilon$ $H^{p}$, then $f=P_{\phi}(f)=P_{\phi}(B \cdot \bar{B} f)=B \cdot P_{\phi}(\bar{B} f)$, showing that $P_{\phi}(\bar{B} f)=\bar{B} f$. For (iii) and (iv) apply Lemma 4 with $K=\{f\}$ and $K=\{B|a| \alpha \in \mathbb{G}\}$, respectively.

Conversely, assume a subspace $M \subset H^{p}$ satisfies (i) -(v). Assume first that $p=2$. Set $\phi=$ g.c.d. $\{B: B$ inner, $B(0)=0, B \in M\}$. By (i) and (iii), the indicated set is nonvacuous, and by (iv), $\phi \in \mathbb{M}$. Let $f \in \mathbb{M}$. We show $f \in H^{2}(\phi)$. If $f=f(0)$ (a constant), then $f \in H^{2}(\phi)$. Otherwise $f-f(0)$ has inner part $\chi$ in $M$ by (ii) and $\chi(0)=0$. By definition of $\phi$, $\bar{\phi}(f-f(0)) \in H^{2}$, whence by (v), $\bar{\phi}(f-f(0)) \in \mathbb{M}$, or $f-f(0) \in \phi \cdot M$. A similar inductive argument shows that $f-\sum_{j=0}^{n}\left\langle f, \phi^{j}\right\rangle \phi^{j} \in \phi^{n+1} \mathbb{M}$ $\left(\langle\cdot, \cdot\rangle\right.$ denotes the inner product for $\left.H^{2}\right)$. Hence

$$
f-P_{\phi}(f)=f-\sum_{j=0}^{\infty}\left\langle f, \phi^{j}\right\rangle \phi^{j}
$$

(the infinite series an $H^{2}$-limit) is an element of $\bigcap_{k=0}^{\infty} \phi^{k} M$. Since the associated Toeplitz operator $T_{\phi}$ is completely nonunitary, $\bigcap_{k=0}^{\infty} \phi^{k} M=$ (0). Hence $f=P_{\phi}(f) \in H^{2}(\phi)$, and $\mathbb{M} \subset H^{2}(\phi)$. Since $\phi^{j} \in \mathbb{M}$ for $j=0,1$, $2, \cdots$, and $\mathbb{M}$ is closed, $\mathbb{M}=H^{2}(\phi)$. 
If $1 \leq p<\infty, f \in H^{p}$ has inner-outer factorization $f=\chi \cdot F$, then the map $f \rightarrow f^{\prime}=\chi \cdot F^{p / 2}$ maps a closed subspace of $H^{p}$ satisfying (i) $-(\mathrm{v})$ onto a closed subspace of $H^{2}$ satisfying (i) $-(\mathrm{v})$. For the case $p=\infty$, note $H^{\infty} \subset H^{2}$, so one can conclude as in the case $p=2$ that $f=P_{\phi}(f)$ for $f \in \mathbb{M}$, whence $\mathbb{M} \subset H^{\infty}(\phi)$. Since $\mathbb{M}$ is weak $-{ }^{*}$ closed and contains $\phi^{j}$ for $j \geq 0, \mathbb{M}=H^{\infty}(\phi)$.

4. Analytic Toeplitz operators. For $F$ an element of $H^{\infty}$, the associated analytic Toeplitz operator $T_{F}$ is defined by

$$
\left(T_{F} f\right)\left(e^{i \theta}\right)=F\left(e^{i \theta}\right) f\left(e^{i \theta}\right) \text { for } f \in H^{2} .
$$

These operators have been much studied and many of their properties are well known (Brown and Halmos [2]).

If $b$ is an inner function which is not a linear fractional transformation, $T_{b}$ is a shift operator of multiplicity greater than 1 , hence $T_{b}$ has nontrivial reducing subspaces [4]. Hence if $F$ is a function of such an inner function $b$, any subspace reducing for $T_{b}$ is also reducing for $T_{F}$, whence $T_{F}$ has nontrivial reducing subspaces. Nordgren [ 8] has conjectured that this is the only time $T_{F}$ has nontrivial reducing subspaces. The main result of this section is

Theorem 2. The following are equivalent:

(I) The Toeplitz operator $T_{F}$ has a nontrivial reducing subspace if and only if $F$ is a function of an inner function which is not a linear fractional transformation.

(II) (i) If $F \in H^{\infty}$ has inner-outer factorization $F=\chi G$ and $\mathbb{M} \subset H^{2}$ reduces $T_{F}$, then $M$ reduces $T_{\boldsymbol{x}}$ and $T_{G}$.

(ii) If $\left\{B_{a}=a \in \mathbb{Q}\right\}$ is a collection of inner functions, $\mathbb{M} \subset H^{2}$ reduces $T_{B_{\alpha}}$ for all $a \in \mathbb{Q}$, then $\mathbb{M}$ reduces $B=$ g.c.d. $\left\{B_{\alpha}=\alpha \in \mathbb{G}\right\}$.

For $F \in H^{\infty}$, set $\mathbb{G}_{F}=\left\{f \in H^{\infty}\right.$ : for any $\mathbb{M}$ reducing $T_{F}, \mathbb{M}$ reduces $\left.T\right\}$. Then $\mathbb{Q}_{F}$ is a subalgebra of $H^{\infty}$, and since weak ${ }^{*}$ convergence in $H^{\infty}$ corresponds to weak convergence of analytic Toeplitz operators, $\mathbb{Q}_{F}$ is weak- ${ }^{*}$ closed.

Lemma 5. For $\phi$ an inner function, $\phi(0)=0, \mathbb{C}_{\phi}=H^{\infty}(\phi)$.

Proof. Since $H^{\infty}(\phi)$ is the weak-* closure of polynomials in $\phi$ and weak-* convergence in $H^{\infty}$ corresponds to weak-operator convergence for the associated Toeplitz operators, one has $H^{\infty}(\phi) \subset \mathbb{Q}_{\phi}$. Conversely, if $T_{f} \in \mathbb{Q}_{\phi}$, since $H^{2}(\phi)$ is a reducing subspace for $T_{\phi}$ and $1 \in H^{\infty}(\phi), f=$ $T_{f}(1) \in H^{\infty}(\phi)$, hence $\mathbb{G}_{\phi} \subset H^{\infty}(\phi)$. 
Lemma 6. (I) is equivalent to

(III) for any $F \in H^{\infty}$, there exists an inner function $\phi$ with $\phi(0)=0$ such that $\mathbb{Q}_{F}=H^{\infty}(\phi)$.

Proof. Assume (III), and suppose $\mathbb{A}_{F}=H^{\infty}(\phi)$ and $T_{F}$ has a nontrivial reducing subspace. Since $\phi \in \mathbb{Q}_{F}, T_{\phi}$ has a nontrivial reducing subspace, whence $\phi$ is not a linear-fractional transformation. Since $F \in H^{\infty}(\phi), F$ is a function of $\phi$.

Conversely, for $F \in H^{\infty}$, let $M$ be the intersection of all subspaces of the type $H^{\infty}(\phi), \phi$ inner with $\phi(0)=0$, containing $F$. Then, by Theorem 1 , $\mathbb{M}=H^{\infty}(b)$, where $b$ is inner, $b(0)=0$, and $F=C_{b}(G)=G(b)$ for some $G \in H^{\infty}$. By the construction it follows that $G$ is not a function of a nontrivial inner function. Hence by (I), $T_{G}$ has no nontrivial reducing subspaces, so the $W^{*}$ algebra generated by $T_{G}$ is all bounded operators on $H^{2}$. In particular if $S: f\left(e^{i \theta}\right) \rightarrow e^{i \theta} f\left(e^{i \theta}\right)$ is the standard shift on $H^{2}$, there exists a sequence of polynomials $P_{n}\left(T_{G}^{*}, T_{G}\right)$ in $T_{G}^{*}$ and $T_{G}$ converging weakly to $S$; since $T_{G}=G(S)$,

$$
S=w \cdot_{n \rightarrow \infty}^{*} \lim _{n} P_{n}\left(G(S)^{*}, G(S)\right) .
$$

Since $T_{b}$, a completely nonunitary isometry, is unitarily equivalent to a direct sum of copies of $S$,

$$
\begin{aligned}
& T_{b}=\underset{n \rightarrow \infty}{w *} \lim _{n} P_{n}\left(G\left(T_{b}\right)^{*}, G\left(T_{b}\right)\right), \text { or } \\
& T_{b}=w \cdot{ }^{*} \lim P_{n}\left(T_{F}^{*}, T_{F}\right),
\end{aligned}
$$

so $T_{b}$ belongs to the $W^{*}$-algebra generated by $T_{F}$. Hence $T_{b}$ has at least the reducing subspaces of $T_{F}$. Since $F=f(b), T_{b}$ cannot have any more, hence $\mathbb{Q}_{F}=\mathbb{Q}_{b}=H^{\infty}(b)$, by Lemma 5 .

Proof of Theorem 2. Combine Lemma 6 with Theorem 1.

5. Concluding remarks. In a related study [3], J. A. Deddens and Tin Kin Wong have made some progress on formulation (II) of the Nordgren conjecture for some special cases.

In a recent preprint, Entire Toeplitz operators, I. N. Baker, J. A. Deddens and J. L. Ullman answer the conjecture in the affirmative if $\phi$ is an entire function.

\section{REFERENCES}

1. A. Beurling, On two problems concerning linear transformations in Hilbert space, Acta Math. 81 (1948), 17pp. MR 10, 381. 
2. A. Brown and P. R. Halmos, Algebraic properties of Toeplitz operators, J. Reine Angew. Math. 213 (1963), 89-102. MR 28 \#3350.

3. J. A. Deddens and Tin Kin Wong, The commutant of analytic Toeplitz operators, Trans. Amer. Math. Soc. 184 (1973), 261-273.

4. P. R. Halmos, Shifts on Hilbert spaces, J. Reine Angew. Math. 208 (1961), 102-112. MR $27 \# 2868$.

5. K. Hoffman, Banach spaces of analytic functions, Prentice-Hall, Englewood. Cliffs, N. J., 1965.

6. M. Loève, Probability theory, 2nd ed., University Ser. in Higher Math., Van Nostrand, Princeton, N. J., 1960. MR 23 \#A670.

7. E. Nordgren, Composition operators, Canad. J. Math. 20 (1968), 442-449. MR 36 \#6961.

8. - Reducing subspaces of analytic Toeplitz operators, Duke Math. J. 34 (1967), 175-181. MR 35 \#7155.

9. G. C. Rota, On the representation of averaging operators, Rend. Sem. Mat. Univ. Padova 30 (1960), 52-64. MR 22 \#2899.

10. J. Ryff, Subordinate $H^{p}$ functions, Duke Math. J., 33 (1966), 347-354. MR 33 \#289.

11. T. P. Srinivasan and Ju-Kwei Wang, Weak *-Dirichlet algebras, Function Algebras (Proc. Internat. Sympos. on Function Algebras, Tulane Univ., 1965), edited by F. Virtel, Scott, Foresman, Chicago, Ill., 1966, pp. 216-249. MR 33 \#6441.

12. B. Sz.-Nagy and C. Foias, Harmonic analysis of operators on Hilbert space, Akad. Kiadó, Budapest, 1970; English transl., North-Holland, Amsterdam; American Elsevier, New York, 1971 . MR 43 \#947.

DEPARTMENT OF MATHEMATICS, VIRGINIA POLYTECHNIC INSTITUTE AND ST ATE UNIVERSITY, BLACKSBURG, VIRGINIA 24061 